\title{
Response to the combination use of pazopanib with olaratumab in a patient with lung-metastatic embryonal rhabdomyosarcoma: a case report
}

\author{
Tuo Xing", Yalei Zhang", Xukai Li, Minzhang Guo, Wenhua Liang, Jianxing He \\ Department of Thoracic Surgery and Oncology, the First Affiliated Hospital of Guangzhou Medical University, State Key Laboratory of Respiratory \\ Disease, National Clinical Research Center for Respiratory Disease, Guangzhou Institute of Respiratory Health, Guangzhou, China \\ \#These authors contributed equally to this work. \\ Correspondence to: Jianxing He, MD, PhD, FACS, FRCS (Eng), AATS member. Department of Thoracic Surgery and Oncology, the First Affiliated \\ Hospital of Guangzhou Medical University, No. 151, Yanjiang Rd., Guangzhou 510120, China. Email: drjianxing.he@gmail.com; Wenhua Liang \\ MD. Department of Thoracic Surgery and Oncology, the First Affiliated Hospital of Guangzhou Medical University, State Key Laboratory of \\ Respiratory Disease, National Clinical Research Center for Respiratory Disease, Guangzhou Institute of Respiratory Health, Guangzhou, China. \\ Email: liangwh1987@163.com.
}

\begin{abstract}
Embryonal rhabdomyosarcoma (ERMS) is associated with a low prevalence, poor prognosis, and limited treatment efficacy. Here, we report a case of a 21-year-old male whose disease relapsed in the thoracic cavity following traditional chemotherapy. The patient received eight sequential cycles of traditional chemotherapy using a combination of the cyclophosphamid + vincristine + doxorubicin hydrochloride liposome (CAV) and etoposide + ifosfamide (IE) regimens. The therapeutic effect of the combination regimen had been worked in short times. After a month, ERMS had relapsed in the whole lung after traditional chemotherapy. The treatment method was changed immediately and the patient received targeted therapy with a combination of pazopanib and olaratumab. The therapeutic effect of the combination regimen was evaluated for a complete response (CR). After two months, CT imaging revealed that most of the metastatic lesions in the lung had disappeared. This is the first case to report the use of pazopanib and olaratumab in relapsed ERMS with a curative effect resulting in a CR. Pazopanib is approved for advanced soft tissue sarcoma (STS) and renal cell cancer. Thus, combining pazopanib with targeted therapy may play an important role and provide a reference for the treatment of relapsed ERMS.
\end{abstract}

Keywords: Embryonal rhabdomyosarcoma (ERMS); lung metastasis; target treatment

Submitted Dec 07, 2019. Accepted for publication Oct 10, 2020.

doi: $10.21037 /$ tlcr-19-644

View this article at: http://dx.doi.org/10.21037/tlcr-19-644

\section{Introduction}

Embryonal rhabdomyosarcoma (ERMS) is a rare category of soft tissue sarcoma (STS) that originates in the mesenchymal tissue. There are three major histologic subtypes of rhabdomyosarcoma $(1,2)$ and the head and neck region represent the most affected site. Surgery is the best method for removing early-stage tumors (3); however, a high risk of recurrence or distant metastasis remains afterward. Despite the demonstrated efficacy of many types of chemotherapy in clinical trials, ERMS has a low objective response rate to cytotoxic drugs and a poor longterm prognosis with short progression-free survival (4). More recently, targeted therapy is being selected by many oncologists as a new treatment approach. Furthermore, immunotherapy has been tested in select sarcoma histotypes with encouraging activity, and has led to further evaluation in combination with immunotherapeutic agents, as well as with chemotherapy and radiation. Pazopanib is a multi-receptor tyrosine kinase inhibitor that primarily targets vascular endothelial growth factor receptors $1-3$ 
(VEGFRs1-3), and is currently approved for the treatment of advanced STS and renal cell cancer (5).

Olaratumab is a monoclonal antibody that recently received approval for the treatment of advanced softtissue sarcomas in combination with doxorubicin. In particular, this combination treatment is recommended for a histological subtype in which anthracycline-containing regimens is appropriate and the disease is not amenable to curative surgery or radiotherapy. Olaratumab specifically inhibits platelet-derived growth factor receptor alpha, leading to the inhibition of tumor cell proliferation, angiogenesis, and metastasis (6).

Here, we report a case of a 21-year-old male whose disease relapsed in the thoracic cavity after receiving traditional chemotherapy. The patient was treated with a combination of pazopanib and olaratumab, and showed a complete response (CR) to this regimen.

We present the following case in accordance with the CARE Reporting Checklist (available at http://dx.doi. org/10.21037/tlcr-19-644).

\section{Case presentation}

A 21-year-old Chinese man arrived at our hospital with a cough, blurred vision, and weight loss of $10 \mathrm{~kg}$ over the last 16 months. Upon physical examination, the patient had a rare disease, termed ERMS.

Sixty months prior, the patient had arrived at The First Affiliated Hospital of Zhongshan University with nasal congestion, rogue, and vision loss. After admission, the patient underwent a series of checks, including routine blood screening, as well as an analysis of liver and kidney function, coagulation, and electrolytes. However, all of these tests were normal. Positron emission tomography $(\mathrm{PET}) /$ computed tomography $(\mathrm{CT})$ revealed a tumor in the right sinus-maxillary sinus-medulla-eyelid that was $5.5 \times 4.3 \times$ $5.6 \mathrm{~cm}^{3}$ in size, with an active metabolism, SUV5.2. The immunohistochemistry results revealed CK7(-), MyoD1(+), Myogenin(+), Desmin(+), TTF-1(-), NapsinA(-), Ki67(+), and CD56(+). Histopathological examination of the specimen revealed it to be ERMS T4NOM0. PET-CT indicated that there were no other sites of metastasis. This disease is not amenable to curative surgery or radiotherapy. Therefore, he and his family accepted the chemotherapy regimen, and underwent approximately eight sequential cycles of chemotherapy. The treatment plan specifically included cyclophosphamide $1.2 \mathrm{~g}$ + vincristine $2 \mathrm{mg}+$ doxorubicin $60 \mathrm{mg}$ for four cycles combined with etoposide
$200 \mathrm{mg}$ + isocyclophosphamide $3 \mathrm{~g}$ for four cycles. Unfortunately, after 2 months, multiple metastatic tumors in the bilateral lung were found by $\mathrm{CT}$ at a local medical institution. To date, the patient has subsequently been undergoing treatment with a combination of pazopanib and olaratumab therapy. The method of administration is described that patients take $800 \mathrm{mg}$ of the pazopanib daily continuously and patient injects olaratumab intravenously for a week, which 21 days per cycle. During treatment, the patient experienced drug-related adverse reactions, such as diarrhea, pain, white hair, vision loss, and nose-bleeding after a week; however, the reactions were tolerable without adjusted doses. After a month, the CT showed that the multiple metastatic tumors in the lung were significantly reduced, or had even disappeared (Figure 1). In addition, there were no severe adverse reactions that appeared during treatment. The patient currently walks about $6 \mathrm{~km}$ daily during the course of treatment.

All procedures performed in studies involving human participants were in accordance with the ethical standards of the institutional and/or national research committee(s) and with the Helsinki Declaration (as revised in 2013). Consent was obtained from relatives of the patient for publication of this report and any accompanying images.

\section{Discussion}

ERMS is an extremely rare tumor with an estimated incidence of 0.45 to 0.69 per 100,000 cases (7). The head and neck region is the most affected site, followed closely by the genitourinary tract, in which approximately $25 \%$ of cases occur (3). Metastatic ERMS has a high mortality rate and poor prognosis. Although surgery is the optimal method for removing early-stage tumors, there remains a high risk of recurrence or distant metastasis afterward. Despite clinical trials demonstrating the efficacy of many types of chemotherapy, ERMS has a low objective response rate to cytotoxic drugs and a poor long-term prognosis with short progression-free survival (4). Therefore, some experts emphasize that the treatment options for this disease differ from other STS systemic chemotherapies, and multidisciplinary combination therapy is recommended (1). Recently, some case reports have shown that Endostar combined with chemotherapy may play a synergistic role; however, further clinical confirmation is required (8). Pazopanib is a multi-receptor tyrosine kinase inhibitor that primarily targets vascular endothelial growth factor receptors and is approved for the treatment of advanced 

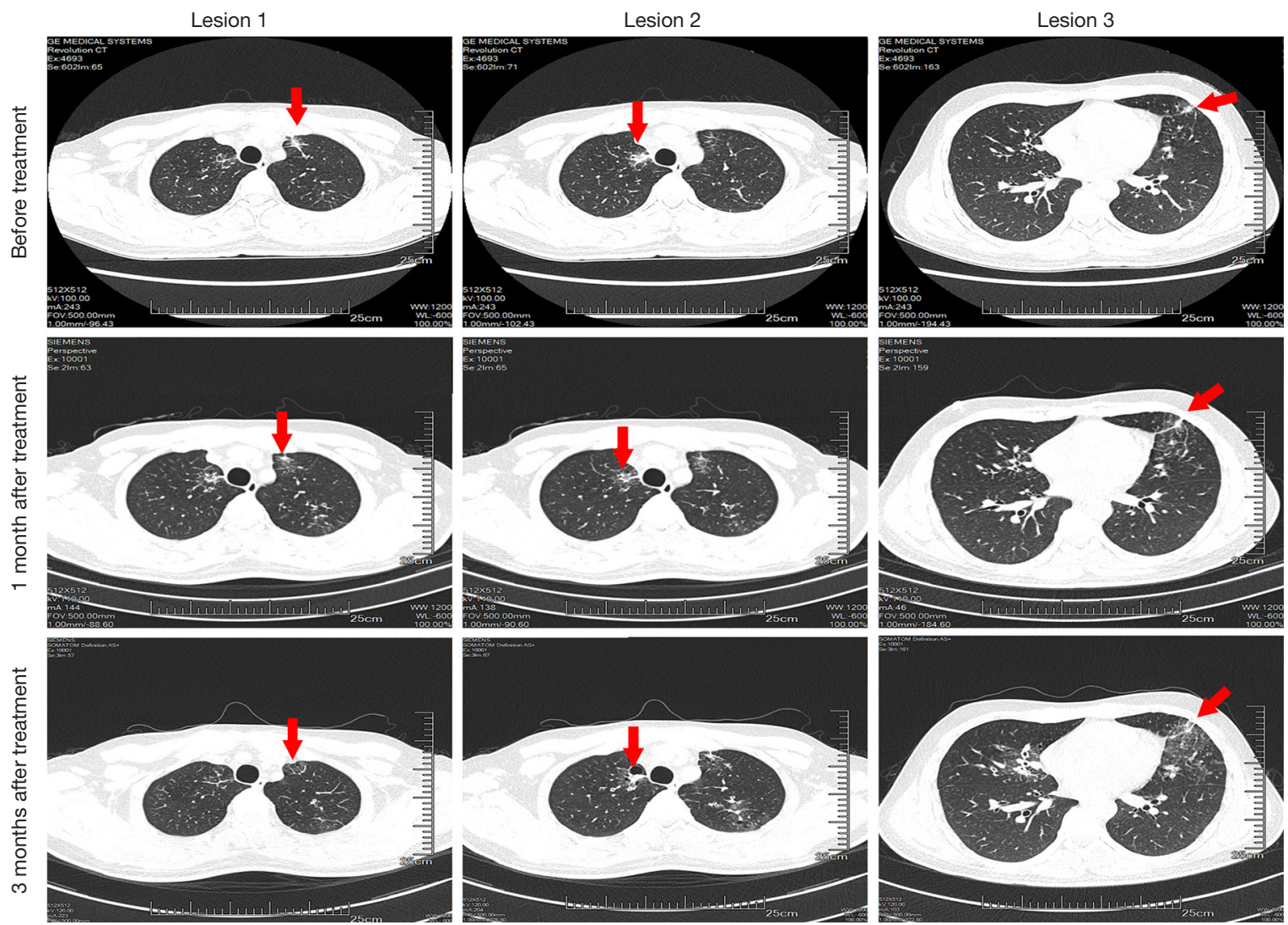

Figure 1 Images before and after treatment. The arrows indicate tumor.

STS and renal cell cancer (5). Moreover, olaratumab is a novel anti-PDGFR- $\alpha$ monoclonal antibody that has been indicated for the treatment of STS in combination with doxorubicin in patients with STS not amenable to curative surgery or radiotherapy. Being one of the only drugs in this setting to be combined with doxorubicin and demonstrate an overall survival benefit (6).

In this study, our patient underwent pazopanib and olaratumab combination therapy after a routine chemotherapy regimen that included cyclophosphamide, vincristine, doxorubicin, etoposide, and ifosfamide for a total of eight cycles. However, after three months, many metastatic tumors in the bilateral lung were identified by CT. The patient subsequently agreed to receive pazopanib combined with olaratumab for further treatment. At the start of therapy, the patient took $400 \mathrm{mg}$ for a week at home. One week later, the patient began taking $800 \mathrm{mg}$ per day. A month later, the patient was injected with olaratumab at the $1^{\text {st }}$ and $8^{\text {th }}$, for total treatment cycle of 21 days. A subsequent $\mathrm{CT}$ review showed that the multiple metastatic tumors in the bilateral lung had disappeared compared to the previous CT results. During treatment, the patient experienced drug-related adverse reactions, including diarrhea, pain, white hair, vision loss, and nose-bleeds after one week; however, these reactions were tolerable without adjusting the doses. While the resistance mechanisms to VEGF inhibitors (e.g., pazopanib) are complex, in this case, we found an obvious treatment effect when it was combined with olaratumab. Therefore, anti-angiogenesis combined with the anti-tumor microenvironment may represent a better treatment choice for such patients with lung-metastatic ERMS. This treatment had a good curative effect, low toxicity, and improved patient prognosis. However, the specific mechanism of action remains unclear and requires further exploration. Oncologists recommend continuing the original regimen, as the patient showed CR after this therapy. Moreover, oncologists would do anything for their patients, including providing a suitable treatment regimen for our patients. Some patients who are not amenable to curative surgery or do not respond to 
conventional chemotherapy may be eligible for pazopanib and olaratumab combined treatment. Therefore, this regimen may play an important role for the treatment of ERMS. The patient agrees with our report very much.

\section{Acknowledgments}

Funding: None.

\section{Footnote}

Reporting Checklist: The authors have completed the CARE Reporting Checklist. Available at http://dx.doi. org/10.21037/tlcr-19-644

Conflicts of Interest: All authors have completed the ICMJE uniform disclosure form (available at http://dx.doi. org/10.21037/tlcr-19-644). WL serves as an unpaid editorial board member of Translational Lung Cancer Research from Apr 2018 to Apr 2021. The other authors have no conflicts of interest to declare.

Ethical Statement: The authors are accountable for all aspects of the work in ensuring that questions related to the accuracy or integrity of any part of the work are appropriately investigated and resolved. All procedures performed in studies involving human participants were in accordance with the ethical standards of the institutional and/or national research committee(s) and with the Helsinki Declaration (as revised in 2013). Consent was obtained from relatives of the patient for publication of this report and any accompanying images.

Open Access Statement: This is an Open Access article distributed in accordance with the Creative Commons Attribution-NonCommercial-NoDerivs 4.0 International License (CC BY-NC-ND 4.0), which permits the non- commercial replication and distribution of the article with the strict proviso that no changes or edits are made and the original work is properly cited (including links to both the formal publication through the relevant DOI and the license). See: https://creativecommons.org/licenses/by-nc-nd/4.0/.

\section{References}

1. Sultan I, Qaddoumi I, Yaser S, et al. Comparing adult and pediatric rhabdomyosarcoma in the surveillance, epidemiology and end results program, 1973 to 2005: an analysis of 2,600 patients. J Clin Oncol 2009;27:3391-7.

2. Lai H, Guo Y, He W, et al. Non-target genetic manipulation induces rhabdomyosarcoma in KrasPtendriven mouse model of ovarian cancer. Transl Cancer Res 2020;9:7458-68.

3. Ruiz-Mesa C, Goldberg JM, Coronado Munoz AJ, et al. Rhabdomyosarcoma in adults: new perspectives on therapy. Curr Treat Options Oncol 2015;16:27.

4. Brennan MF, Alektiar KM, Maki RG. Cancer: Principles \& Practice of Oncology. New York: Lippincott Williams \& Wilkins; 2011.

5. Subbiah V, Meyer C, Zinner R, et al. Phase Ib/II Study of the Safety and Efficacy of Combination Therapy with Multikinase VEGF Inhibitor Pazopanib and MEK Inhibitor Trametinib In Advanced Soft Tissue Sarcoma. Clin Cancer Res 2017;23:4027-34.

6. Moore DC, Lavery LA. Olaratumab: A New Strategy in the Treatment of Advanced Soft-Tissue Sarcoma. J Adv Pract Oncol 2018;9:235-40.

7. Van Gaal JC, De Bont ES, Kaal SE, et al. Building the bridge between rhabdomyosarcoma in children, adolescents and young adults: the road ahead. Crit Rev Oncol Hematol 2012;82:259-79.

8. Han T, Chen J, Luan Y, et al. Successful treatment of relapsed testicular embryonal rhabdomyosarcoma with Endostar and traditional chemotherapy: a case report. Onco Targets Ther 2018;11:5287-91.
Cite this article as: Xing T, Zhang Y, Li X, Guo M, Liang W, He J. Response to the combination use of pazopanib with olaratumab in a patient with lung-metastatic embryonal rhabdomyosarcoma: a case report. Transl Lung Cancer Res 2021;10(1):483-486. doi: 10.21037/tlcr-19-644 